\title{
THERMAL CHANGES IN THE HARD DENTAL TISSUE AT DIODE LASER ROOT CANAL TREATMENT
}

\author{
Ts. Uzunov ${ }^{1}$, R. Grozdanova ${ }^{1}$, E. Popova ${ }^{1}$ and T. Uzunov ${ }^{2}$ \\ ${ }^{1}$ Department of Conservative Dentistry, Section of Dental Physical Therapy, Faculty of Dental Medicine, \\ Medical University - Sofia \\ ${ }^{2}$ Department of Prosthetic Dentistry, Faculty of Dental Medicine, Medical University - Sofia
}

\begin{abstract}
Summary. The laser coagulation at the apical part of the root canal after vital extirpation is a proper method of preventing complications such as pain, bleeding, remaining vital pulp. The aim of the present survey is to register the thermal changes that occur on the tooth surfaces during laser treatment of the root canal after vital extirpation. An in vitro study of 30 extracted teeth has been conducted. The teeth have been prepared with ProTaper nickel-titanium machine tools and wiped dry. During the course of laser treatment of root canals with a diode laser DenLase temperatures, changes of the surface of the hard dental tissues have been recorded with infrared camera FLIR T330. The captured thermal images have been processed with software product Flir Reporter Pro 9. In conclusion, temperature changes in hard dental tissues at diode laser treatment of the root canal are biocompatible.
\end{abstract}

Key words: diode laser radiataion, temperature changes, infrared thermography

\section{INTRODUCTION}

$\mathrm{T}$ he use of lasers in endodontics for root canal treatment after vital nerve extirpation is an effective method for preventing the possible complications - pain, vital pulp remainings, restpulpitis, bleeding. The diode laser with a wavelength of $810 \mathrm{~nm}$ at different impulse regiments leads to apical coagulation that reduces the risk of possible complications to emerge [1, 2, 3].

The laser releases a large amount of energy that is transformed into heat and beside the apical coagulation the hard dental tissues can be damaged. Overheating of the dentinal walls and periapical tissues can lead to irreversible changes - large 
areas of melting, recrystallization of the mineral matrix, surface microfractures and carbonization $[4,5]$. Change of temperature on the outer root surface and apex with $7-8^{\circ} \mathrm{C}$ does not lead to periodontal damage but temperature rise more than $10^{\circ} \mathrm{C}$ can damage the surrounding bony structures $[6,7]$.

Infrared thermography proved to be quite precise method for temperature registration in the maxillo-facial area $[8,9,10]$. It has been proven to be effective in the focal diagnostics $(9,10,11)$ and can also be used in experimental studies to register precise temperature changes during laser radiation $[3,5,6]$.

The aim of the present survey is to register the thermal changes that occur on the tooth surfaces during laser treatment of the root canal after vital extirpation.

\section{MATERIAL AND METHODS}

An in vitro study with 30 freshly extracted single rooted teeth has been conducted. External root surfaces have been cleaned with a periodontal curette. Root canals of all teeth have been prepared by crown-down technique with machine nickel-titanium instruments - ProTaper Universal (Dentsply Maillefer) with a final file F3. An alternating rinsing with $3 \% \mathrm{H} 2 \mathrm{O} 2,2.5 \% \mathrm{NaOCl}$ and distilled water has been conducted as well as $17 \%$ EDTA gel. The root canals have been dried up with sterile paper points.

The survey has been conducted in thermally controlled environment without sources of heating or cooling - air conditioned room at $22^{\circ} \mathrm{C}$. The prepared tooth has been fixed by a paper stand. The operator held the paper stand with the one hand and the laser tip with the other.

A diode laser system "DenLase" with wavelenght $810 \mathrm{~nm}$ and optic fiber with

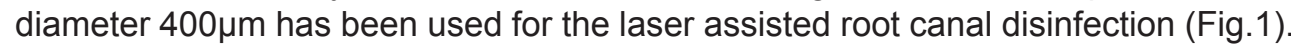

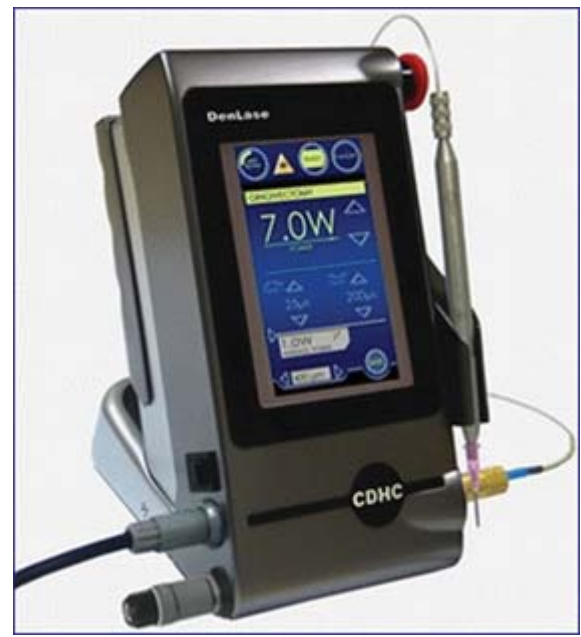

Fig. 1. Laser unit "DenLase" 
The laser treatment has been conducted at impulse regimen with power $2 \mathrm{~W}$ at pulse length $1 \mathrm{~ms}$ and pulse interval $1 \mathrm{~ms}$, so the average power has been $1 \mathrm{~W}$.

The tip of the fiber optic was inserted into the root canal to the apical stop and was drawn about $1 \mathrm{~mm}$ backwards, and then the laser radiation was initiated. The fiber optic was withdrawn from apical to coronal with slow circular motion within 8 seconds (Fig. 2, 3, 4).
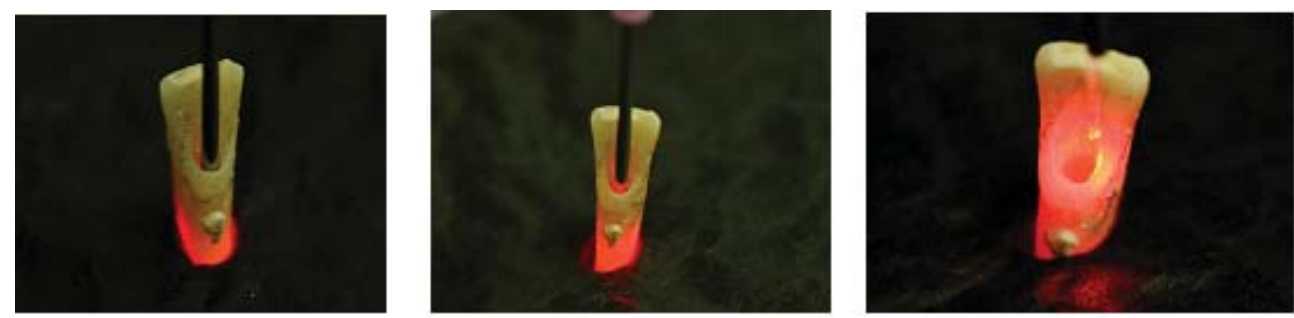

Fig. 2, 3, 4. Laser treatment of the root canal - at the apex, middle part and the crown

Temperature changes on the surface of the hard dental tissues and the apex of the tooth were captured with two thermal shots - in the beginning and in the end of the laser radiation - with an infrared camera Flir T330.

The captured thermal images have been processed with software product Flir Reporter Pro 9.

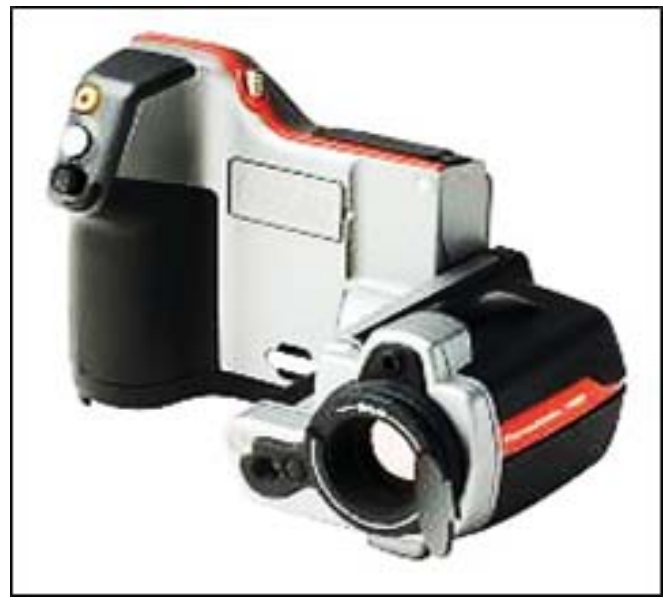

Fig. 5. Infrared camera Flir T330 


\section{RESULTS AND CONCLUSION}

The registered temperature changes in the apex and the outer root surface were within $8^{\circ} \mathrm{C}$ at 22 from 30 investigated teeth; at 5 teeth deviations ranged from $2^{\circ} \mathrm{C}$ to $4^{\circ} \mathrm{C}$; in 3 cases there was a dramatic increase in temperature (Fig. 6).

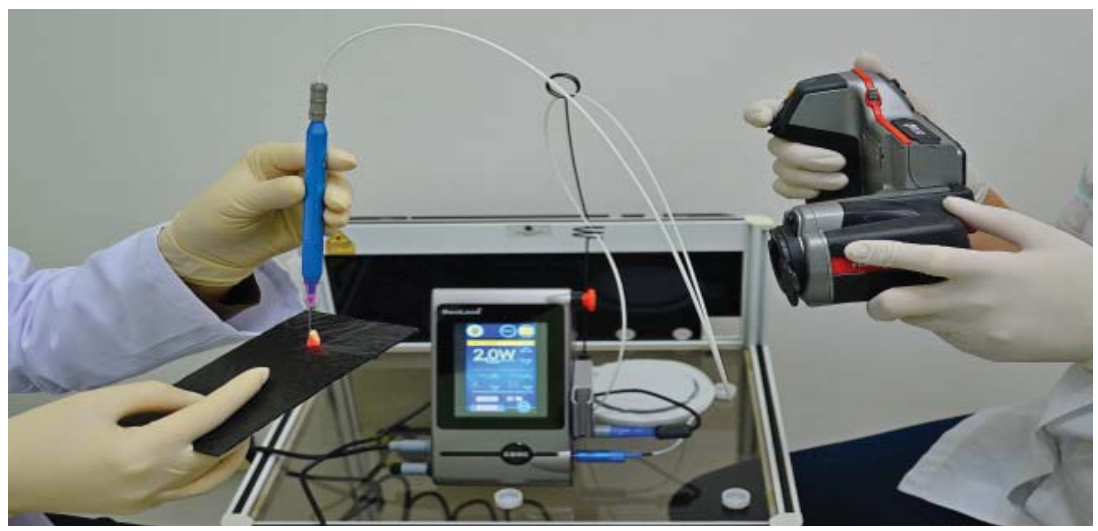

Fig. 6. Design of the investigation

The increase in the temperature at the root surface is dependent on the thickness of the root walls. When teeth were with a massive root (upper central incisors, bicuspids) the variation was small, whereas in teeth with thin roots the variation was greater. The thickness of the root canal wall depends on the degree of preparation. When over instrumentation occurred smaller size of hard dental tissue remained and this led to a temperature increase. Teeth with abnormal $2-4^{\circ} \mathrm{C}$ were three lateral incisors and 2 upper premolars and final treatment with $\mathrm{F} 3$ file led to the withdrawal of a large amount of hard dental tissue from the root.

During treatment, a possible activation of the tip of the fiber optic by coagulated pulp debris can occur, which leads to significant increase in temperature, in our survey 3 cases. When the tip of the fiber optic was activated by mistake, it was cleaned or trimmed.

Temperature changes in the apex and outer root surface during laser treatment of the root canal using a diode laser are biocompatible.

\section{REFERENCES}

1. B e e r, F. et al. The influence of water/air cooling on collateral tissue damage using a diode laser with an innovative pulse design (micropulsed mode)-an in vitro study. - Lasers. Med. Sci., 28, 2013, № 3, 965-971.

2. Faria, M. I. et al. Effects of 980-nm diode laser on the ultrastructure and fracture resistance of dentine. - Lasers Med Sci., 28, 2013, № 1, 275-280. 
3. Marchesan, M. A. et al. Ultrastructural analysis of root canal dentine irradiated with 980-nm diode laser energy at different parameters. - Photomed. Laser. Surg., 26, 2008, № 3, 235-240.

4. Alfre do, E., et al. Temperature variation at the external root surface during $980-\mathrm{nm}$ diode laser irradiation in the root canal. - J. Dent., 36, 2008, № 7, 529-534.

5. da Fonseca Alvarez, A. et al. Temperature changes on the root surfaces of mandibular incisors after an 810-nm high-intensity intracanal diode laser irradiation. - J. Biomed. Opt., 17, 2012.

6. da Costa Ribeiro, A. et al. Effects of Diode Laser $(810 \mathrm{~nm})$ Irradiation on Root Canal Walls: Thermographic and Morphological Studies. - J. Endod., 33, 2007, № 3, 252-255.

7. Hussey, D. L. et al. Thermo-graphic assessment of heat generated on the root surface during post space preparation. - Int. Endod. J., 30, 1997, № 3, 187-190.

8. Grozdan ova, R. et A. Kisselova. Thermography - new method in the oral diagnostics. -16 Annual Assembly of IMAB, 4-7.05.2006, Varna, OP.

9. Grozdanova, R. Thermodiagnostics of focal infection in the maxillo-facial region. Disertation, Sofia, 2012, 191.

10. Grozdanova, R., A. Kisselova. Thermography - essence and aplication in the diagnostics of the focal infection with origin from the maxillo-facial area. - Dental Medicine Problems, 38, 2012, part II, 36-44.

11. Grozdanova, R. Thermodiagnostics of focal infection in the maxillo-facial region. Allergology and Oral Medicine: principles and practices. Sofia. 2013, 220-228.

Corresponding author:

R. Grozdanova

Faculty of Dental Medicine

Medical University

1 Sv. G. Sofiyski st.

BG - 1431 Sofia

e-mail: grozdanova_raia@yahoo.com 